\title{
EXPERIMENTAL DATA FOR INFLUENCE OF GROWTH HORMONE AND SOMATOSTATIN ON PLASMA FIBRINOLYTIC ACTIVITY IN RATS
}

\author{
N. Negrev, Y. Nyagolov*, E. Stanchewa \\ Department of Physiology, Medical University, Varna, Bulgaria, *Department of Physiology, \\ Medical University, Sofia, Bulgaria
}

Reviewed by: Assoc. prof. K. Hristozov, PhD

SUMMARY

Background: Somatotropin (growth hormone) and somatostatin perform various functions in living organisms. Special point of interest is participation of these hormones in haemostasis. Aim: This study was focused on possible involvement of somatotropin and somatostatin in the endocrine regulation of fibrinolytic system. Materials and methods: Expriments were carried out on 168 male Wistar rats. Blood samples were gathered by cardiac puncture under ether narcosis. Some basic parameters of plasma fibrinolytic activity were measured using Boehringer Mahnnheim and Behring tests on Schnitger und Gross coagulometer. Results and discussion: Somatotropin $(0.2 \mathrm{mg} / \mathrm{kg}$ b.m.) applied s.c. for three consecutive days decreased plasminogen $(p<0.001)$ and euglobulin clot lysis time $(p<0.001)$; increasedand $? 2$-antiplasmin $(p<0.001)$ and plasma kallikrein activity $(\mathrm{p}<0.001)$. Somatostatin $(0.1 \mathrm{mg} / \mathrm{kg}$ b.m.) applied alone, as well as somatostatin applied 3 hours before somatotropin in the same dose and scheme, increased plasminogen $(p<0.001)$, its activators $(p<0.001)$ and fibrinogen/fibrin degradation products, while ?2-antiplasmin and plasma kallikrein activity were significantly reduced $(p<0.001)$. The significant changes of plasma kallikrein activity by both hormones and their combined application did not correlate with the shifts of plasma fibrinolytic activity. Conclusion: These results suppose the two hormones are substantial regulators of the fibrinolytic system, as somatotropin suppresses, while somatostatin stimulates plasma fibrinolytic activity.

Keywords: somatotropin, somatostatin, fibrinolysis, hemocoagulation, plasminogen

\section{INTRODUCTION}

Fibrinolytic system is an essential enzyme defense system operating in delicate balance with hemocoagulation and anticoagulation systems (14). Research interest towards physiological or impaired fibrinolysis is constantly increasing because of the existence of abundance of acquired and congenital fibrinolytic disorders, occasionally related to widespread significant social diseases as atherosclerosis, diabetes, malignancies, etc. $(4,10,11)$. Moreover, recent extensive implication of thrombolytic therapy in clinical practice increases the importance of this issue, having in mind the considerable controversy existing about presently available thrombolytic agents (3). All these considerations, from scientific and practical point of view determine the research interest on regulation of fibrinolytic system.

Endocrine system undoubtedly plays an important role in fibrinolysis regulation and various hormones are involved in these processes $(9,12,17,19)$. However, data about con-

Address for correspondence:

Y. Nyagolov, Department of Physiology, Medical University, 2 Zdrave

Str., 1431, Sofia, BULGARIA tribution of hypothalamic-pituitary growth hormone axis in relation to fibrinolysis are relatively limited and insufficiently defined. This study was designed to reveal the effects of somatotropin - growth hormone (GH) and somatostatin - somatotropin releasing inhibitor hormone (SRIH) on some basic parameters of fibrinolytic system in rats.

\section{MATERIALS AND METHODS}

\section{Animals}

Experiments were performed on 168 male Wistar rats weighing 200-220 g, housed at optimal conditions, exposed to free access to standard chow and water in accordance with the international and local legislation. Twelve of the animals were sacrificed as a source for standard plasma. Number of animals was higher, because the necessary blood volume for the experiments could not be received from a single rat.

Design of hormone administration

Test animals were divided into four equal groups. Hormones were applied subcutaneously once daily for three 
consecutive days. First group was injected by GH (Somatotropin human, Boehringer Mannheim, activity 1.80 units $/ \mathrm{mg}$ ) $0.2 \mathrm{mg} / \mathrm{kg}$ body mass, second group - by SRIH (Tyr-somatostatin, Sigma, minimum $97 \%$ ) $0.1 \mathrm{mg} / \mathrm{kg}$ body mass, third group - by the two hormones using the same procedure, as SRIH was administered three hours before GH. The doses of the hormones were determined experimentally by examination of logarithmic dose-effect dependence and according to literature data (2). Fourth group was injected by saline (the solvent of the hormones) by the same scheme and volume per $\mathrm{kg}$ body mass.

Blood collection and procedure for plasma acquisition The necessary blood volume from a rat was acquired by a cardiac puncture under ether narcosis in plastic syringes. Sodium citrate $(0.11 \mathrm{~mol} / \mathrm{L})$ was used as anticoagulant. Citrated blood was centrifuged for $10 \mathrm{~min}$ at $2000 \mathrm{~g}$ and plasma received was stored at 4 degrees centigrade. The parameters examined were determined not later than 2 hours. Only for fibrinogen/fibrin degradation products (FDP) measurement serum was used, received in the original test-tubes of the test.

\section{Determination of fibrinolysis parameters}

The euglobulin clot lysis time (ECLT) was determined by a routine method using test of National Institute of Hematology and Transfusiology, Sofia (1). Clotting times were measured by coagulometer of Schnitger und Gross. Plasminogen (PL) and ?2-antiplasmin (?2-AP) biological activities were evaluated spectropfotometrically using respectively Boeringer Mannheim and Behring tests, as absorbencies were measured at $250 \mathrm{C}$ and $?=405 \mathrm{~nm}$. Plasma kallikrein activity was estimated by Boeringer Mannheim test. FDP were measured by a semiquantitative Boeringer Mannheim test.

\section{Hystological studies}

Slices of the internal organs of all rats were stained by hematoxylin/eosin and for fibrin by Weigert and were examined for possible haemorrhages and/or intravascularal coagulation.

\section{Statistical analysis}

Data were analyzed by variation analysis using unpaired two tailed T-test. Values of P less than 0.05 were accepted to be significant. Percent deviation (?\%) was calculated compared to the results of the control groups accepted for zero.

\section{RESULTS}

Euglobulin clot lysis time (Fig.1) was increased significantly by GH by $70.13 \%$ (?\%) compared to control group. SRIH alone and in combination with GH shortened this variable respectively by $43.96 \%(\mathrm{p}<0.001)$ and $45.26 \%$ $(\mathrm{p}<0.001)$.
GH increased plasma kallikrein activity (Fig. 2) by $82.41 \%$ $(\mathrm{p}<0.001)$. SRIH alone and GH after SRIH pre-treatment

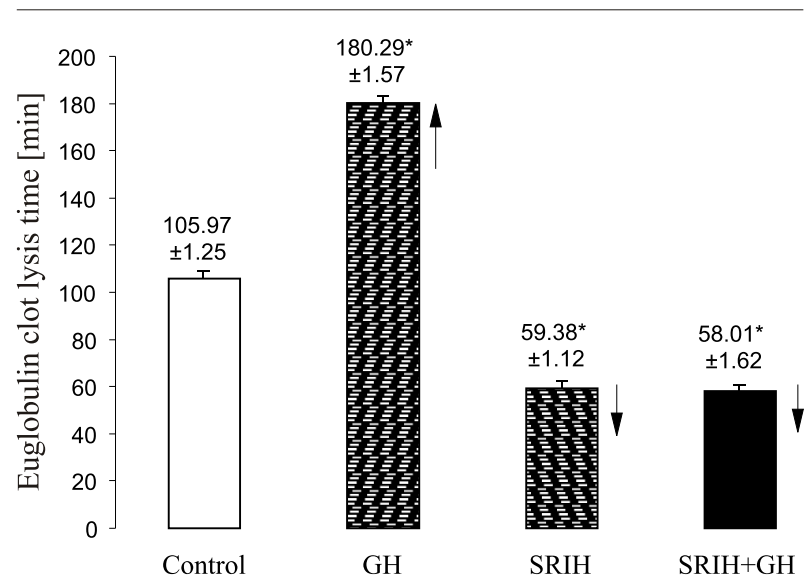

Fig. 1: Effect of growth hormone (GH), somatostatin (SRIH), and GH after 3 hours SRIH pretreatment $(S R I H+G H)$ on euglobulin clot lysis time after 3 days s.c. application. Data are presented as mean \pm S.E.M. Arrows indicate increase/decrease.

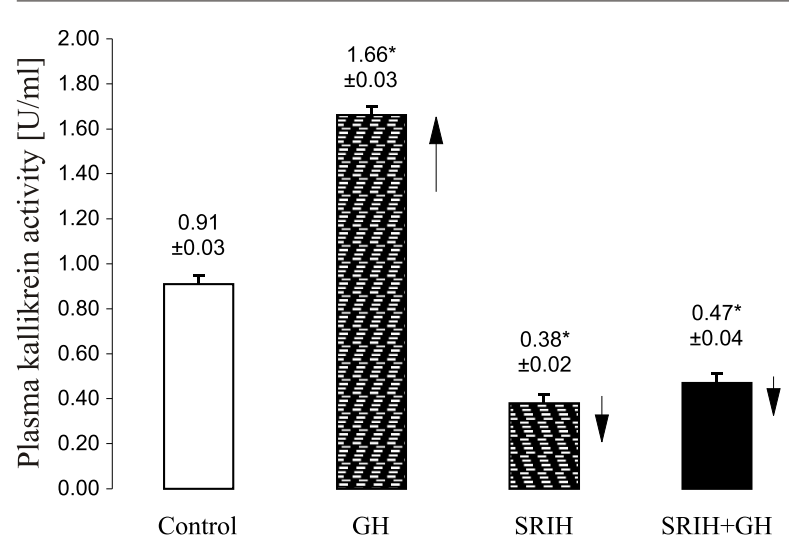

Fig. 2: Effect of growth hormone (GH), somatostatin (SRIH), and GH after 3 hours SRIH pretreatment $(S R I H+G H)$ on plasma kallikrein activity after 3 days s.c. application. Data are presented as mean \pm S.E.M. Arrows indicate increase/decrease.

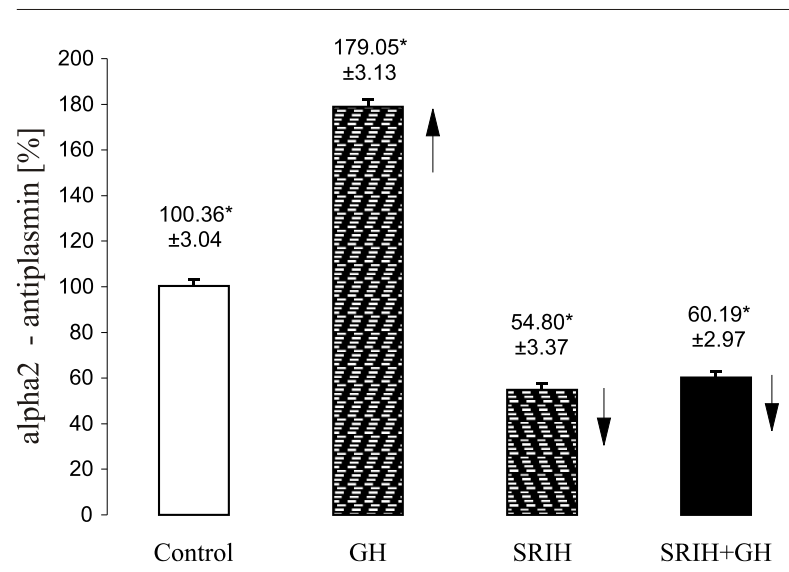

Fig. 3: Effect of growth hormone (GH), somatostatin (SRIH), and GH after 3 hours SRIH pretreatment $(S R I H+G H)$ on alpha2antiplasmin after 3 days s.c. application. Data are presented as mean \pm S.E.M. Arrows indicate increase/decrease. 
decreased this index respectively by $58.24 \%(\mathrm{p}<0.001)$ and by $48.35 \%$ ( $<<0.001)$.

GH significantly increases plasma ?2-AP biological activity (Fig. 3) by $78.40 \%(\mathrm{P}<0.001)$ and decreases PL activity (Fig. 4) by $64.89 \%(\mathrm{P}<0.001)$. After SRIH administration ?2-AP activity is diminished by $45.39 \%(\mathrm{P}<0.001)$, but $\mathrm{PL}$ activity is elevated by $56.66 \%(\mathrm{P}<0.001)$. GH after $3 \mathrm{~h}$ SRIH pre-treatment demonstrates suppression of ?2-AP activity by $40.02 \%(\mathrm{P}<0.001)$ and increase of PL activity by $67.75 \%(\mathrm{P}<0.001)$.

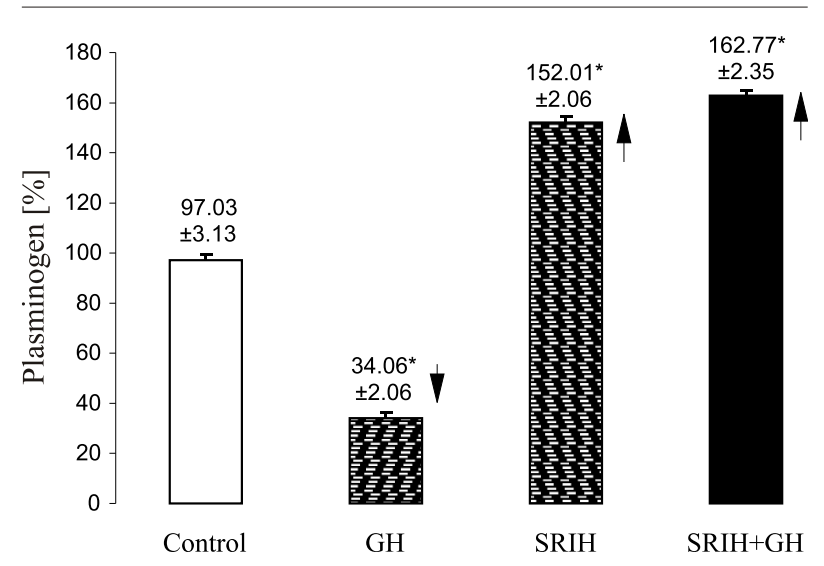

Fig. 4: Effect of growth hormone (GH), somatostatin (SRIH), and GH after 3 hours SRIH pretreatment $(S R I H+G H)$ on plasminogen after 3 days s.c. application. Data are presented as mean \pm S.E.M. Arrows indicate increase/decrease.

The FDP content was less than 10 ?g/ml in control and GH treated groups, wile SRIH alone and in combination with GF elevated FDP between 10 and 40 ?g/ml.

Macroscopic inspection and histological slices of internal organs in all groups of rats did not show signs of haemorrhages or intravascular coagulation.

\section{DISCUSSION}

Our attention was attracted by somatotropin and somatostatin, not only because information about participation of these two hormones in hemocoagulation and fibrinolysis is relatively scarce $(8,13)$, but also because of the fact that some of their basic effects are realized in the liver $(16,18)$, where the considerable amount of substrates and enzymes engaged in fibrinolysis and hemocoagulation is produced (14). SRIH on the one hand is an essential regulator of GH episodic secretion (6), on the other hand it is reported to be present in hepatic nerves as a neurotransmitter (5), parallel to its role as a gastrointestinal hormone. Moreover evidences about expression of GH and SRIH receptors (binding sites) in hepatocytes are abundant. Having in mind all these facts, the results of this study were not unanticipated.

ECLT was significantly increased ( $\mathrm{P}<0.001)$ by GH (Fig. 1). This assay is widely used classical criterion for plasminogen activators (1), and we may accept ST reduces the activity and/or content of these activators. As far as plasma kallikrein activity is a possible intrinsic plasminogen activator, its measurement in this study was reasonable. However, the increased plasma kallikrein activity in the group of GH treated rats (Fig. 2) is not in good harmony with the previous finding of increased ECLT - a sign for suppressed fibrinolytic activators. In this study we have not measured the basic physiological plasminogen activators - tissue type plasmonogen activator (TPA) and urokinase, and from these results it is difficult to assume where the basic GH effect is performed. These data allow us to accept as most probable the hypothesis that not always a positive correlation exists between plasma kallikrein activity and plasma fibrinolytic activity, which is supported by literature data (14). Moreover, ECLT is dependent not only on fibrinolysis activators, but also on the content of fibrinogen, plasminogen and plasmin.

Significant plasminogen suppression in GH treated rats (Fig. 4) reveals possibility for involvement of $\mathrm{GH}$ in plasminogen activity regulation. Lowered plasminogen by itself to a certain extent is a sign of suppressed plasma fibrinolytic activity. Having in mind the fact that plasminogen is synthesized mainly in the liver, it may be hypothesized GH performs its suppressing effect predominantly here. Data for presence of GH receptors in the liver and reports for some GH suppressive effects here (15) are in support of this presumption.

The shifts of ?2-AP, which is not only a main plasmin inhibitor, but also lowers to certain extent plasminogen level (14), harmonize well with plasminogen findings. As far as ?2-AP is produced mainly in the liver (1), the hypothesis that ST performs its effect by a stimulation of ?2-AP synthesis is very probable. Plenty of literature data for stimulating effects of $\mathrm{GH}$ in the liver are available (6).

The absence of significant shifts of FDP in the experiments is a logical consequence of the already presented results: decreased plasminogen (Fig. 4) and its activators (Fig. 1) and increase of ?2-AP (Fig. 3).

Analysis of the results of SRIH treated animals show all measured parameters are significantly $(p<0.001)$, but controversially shifted in comparison with those treated by $\mathrm{GH}$ (Figg. 1,2,3 and 4). The considerable increase of plasminogen and its activators, as well as the diminished ?2-AP, may be interpreted as a specific evidence for increased plasma fibrinolytic activity by this hormone.

Having in mind FDP are essential criterion for plasma fibrinolytic activity, their increase may be interpreted as an indication of stimulation of this activity by SRIH.

The significant reduction of plasma kallikrein activity by SRIH (Fig. 2) is an important finding. It is obvious that plasma kallikrein activity in both experimental and GH treated groups would not be assumed as a factor defining plasma fibrinolytic activity. Literature data allow suggestion that stimulating effect of SRIH of plasma fibrinolytic activity is probably mediated by another activator of this system (7).

As far as the site and mechanism(s) of the action of SRIH are concerned, the data of this study allow some speculations. On the one hand GH is found to suppress signifi- 
cantly plasma fibrinolytic activity, while SRIH stimulates it. On the other hand SRIH is a basic inhibitor of GH secretion (6). On this base, though very hypothetically, may be supposed that at least part of the phenomena observed are related to suppressed GH secretion. Nonetheless, possibility for SRIH to perform direct effect on hepatic biosynthesis of plasminogen and/or ?2-AP would not be neglected. Plenty of data about various metabolic effect of SST are available recently (16).

Striking is the observation that the results of the third group of animals treated by GH after SRIH pretreatment reproduce the results of only SRIH treated rats. (Figg. 1, 2, 3 and 4). This is an illustration of a significantly increased plasma fibrinolytic activity. These data may be interpreted as an evidence for an eliminated effect of the exogenous GH (and may be of endogenous $\mathrm{GH}$ ) on plasminogen-dependent fibrinolysis by SRIH.

In summary, the data received allow to assume GH and SRIH are important factors for plasma fibrinolytic activity. GH inhibits and reduces significantly plasminogen and its activators and increases ?2-AP. SRIH applied alone or with a following treatment by $\mathrm{GH}$ demonstrate opposite effects: increase of plasminogen and its activators and on this base FDP, while ?2-AP is significantly reduced. Shifts of plasma kallikrein activity observed do not correlate with plasma fibrinolytic activity.

In relevance to increased recent use of GH and SRIH in clinical practice the results of this study may be considered for further detailed investigations of the effect of these hormones on fibrinolytic system in clinical conditions.

\section{REFERENCES}

1. Лисичков, Т. 3. Нарушения на хемостазата. София, Медицина и Физкултура, 1987, 32-36.

2. Bak, B. Fracture healing and growth hormone. A biochemical study in the rat. Danish Medical Bulletin. 1993, 40, 519-536.

3. Collen D., H.R. Lijnen. Thrombolytic agents.Thromb. Haemost. 2005, 93(4):627-30.

4. Dunn E.J., P.J. Grant. Type 2 diabetes: an atherothrombotic syndrome.- Curr. Mol. Med. 2005, 5(3):323-32.

5. Feher, E., M. Fodor, J. Feher. Ultrastructural localization of somatostatin- and substance P-immunoreactive nerve fibres in the feline liver.Gastroenterology, 1992, 102, 287-294.
6. Frago, L.M., J.A. Chowen. Basic physiology of the growth hormone/insulin-like growth factor axis.Adv. Exp. Med. Biol. 2005, 567:1-25

7. Francis, R.B. Jr. Clinical disorders of fibrinolysis.Blut, 1989, 59, 1-14.

8. Ingerslev, J., P.D. Christensen, S. Stenbjerg, N. Moller, etc. Diabetes like alterations in hemostatic parameters after growth hormone administration for one week in normal man.- Journal of Diabetic Complications, 1989, 3, 103-106.

9. Juan-Vague I., M.C. Alessi, P.E. Morange. Hypofibrinolysis and increased PAI-1 are linked to atherothrombosis via insulin resistance and obesity.Ann. Med. 2000, 32 Suppl 1:78-84.

10. Kannel W. B. Overview of hemostatic factors involved in atherosclerotic cardiovascular disease.Lipids, 2005, 40(12):1215-20.

11. Kimura K., M. Kosuge. Fibrinolysis in patients with acute coronary syndrome.- Nippon Rinsho. 2006, 64(4):701-8.

12. Klein M., F. Darbe, P. Kaminsky, M. Duc. Les troubles de 1,hemostase dans les dysthyroidities.Pathologie Biologie, 1993, 41, 260-275.

13. Mielke, C. H. Jr., R. Rodvien. Somatostatin: influence on hemostasis.- Methabolism: Clinical and Experimental, 1978, 27: 1369-1375.

14. Mosesson M. W. Fibrinogen and fibrin structure and functions.- J. Thromb. Haemost., 2005, 3(8):1894-904.

15. Murayama, N., M. Chimada, Y. Yamazoe, R. Kato. Difference in the susceptibility of two phenobarbital inducible forms, P450IID1 and P450IID2 to thyroid hormone and growth hormone induced suppression in rat liver; phenobarbital inducible P450IID2 suppression by thyroid hormon acting directly, but not through the pituitary system.- Molecular Pharmacology, 1991, 39, 811-817.

16. Panteris V, D. G. Karamanolis. The puzzle of somatostatin: action, receptors, analogues and therapy.- Hepatogastroenterology. 2005;52:1771-81.

17. Tsantarliotou M., Taitzoglou I., Kokolis N. The effect of dexamethasone on tissue fibrinolytic system in male and female rats.- In Vivo. 1999, 13(2):119-24.

18. Vergani, G., A. Mayerhofer, A. Bartke. Acute effects of human growth hormone on liver cells in vitro: a comparison with livers of nice for human growth hormone.- Tissue \& Cell, 1991, 23, 607-612.

19. Yang, X.C., T.Y. Jing, L.M. Resnick, G.B. Phillips. Relation of hemostatic risk factors to other risk factors for coronary heart disease and to sex hormones in men.- Arteriosclerosis and Thrombosis, 1993, 13: 467-471. 